\title{
Digital Control of Plasma Position in Damavand Tokamak
}

\author{
M. Emami, A. R. Babazadeh, M. V. Roshan, M. Memarzadeh, and H. Habibi \\ Plasma Physics Lab., NFRC, AEOI, Iran
}

Received on 26 June, 2001

\begin{abstract}
Plasma position control is one of the important issues in the design and operation of tokamak fusion research device. Since a tokamak is basically an electrical system consisting of power supplies, coils, plasma and eddy currents, a model in which these components are treated as an electrical circuits is used in designing Damavand plasma position control system. This model is used for the simulation of the digital control system and its parameters have been verified experimentally. In this paper, the performance of a high-speed digital controller as well as a simulation study and its application to the Damavand tokamak is discussed.
\end{abstract}

\section{Introduction}

Tokamak is an electrical system consisting of a transformer, plasma, eddy currents, power supplies and windings [1]. The changes in the plasma parameters such as density, temperature and current create forces, which moves the plasma from its equilibrium position. Plasma position feedback control in tokamaks via the application of classical or modern control theory has been studied extensively. Most tokamaks have adopted analog controllers. The rapid development of digital signal processors (DSP) has increased the speeds to such an extent which digital controllers have become more attractive [2]-[6].

This paper describes the control system simulation based on a high speed Texas- Instrument DSP and its application to the plasma position control in Damavand tokamak.

\section{Damavand Plasma Position Model}

Damavand is a tokamak with an elongated plasma cross-section [7]. The cross-section of Damavand is shown in Fig. 1 and its parameters are given in Table 1. Several forces are acting on the plasma; these are the expansion force due to the plasma pressure, the forces due to the interactions of magnetic field of vacuum vessel eddy currents and the external magnetic field with the plasma itself. In Damavand, the control coils, primary coils, vacuum vessel and plasma that form several mutually interacting electric circuits, generate these forces. Their applied voltages, resistance, and self and mutual inductance can describe these circuits.

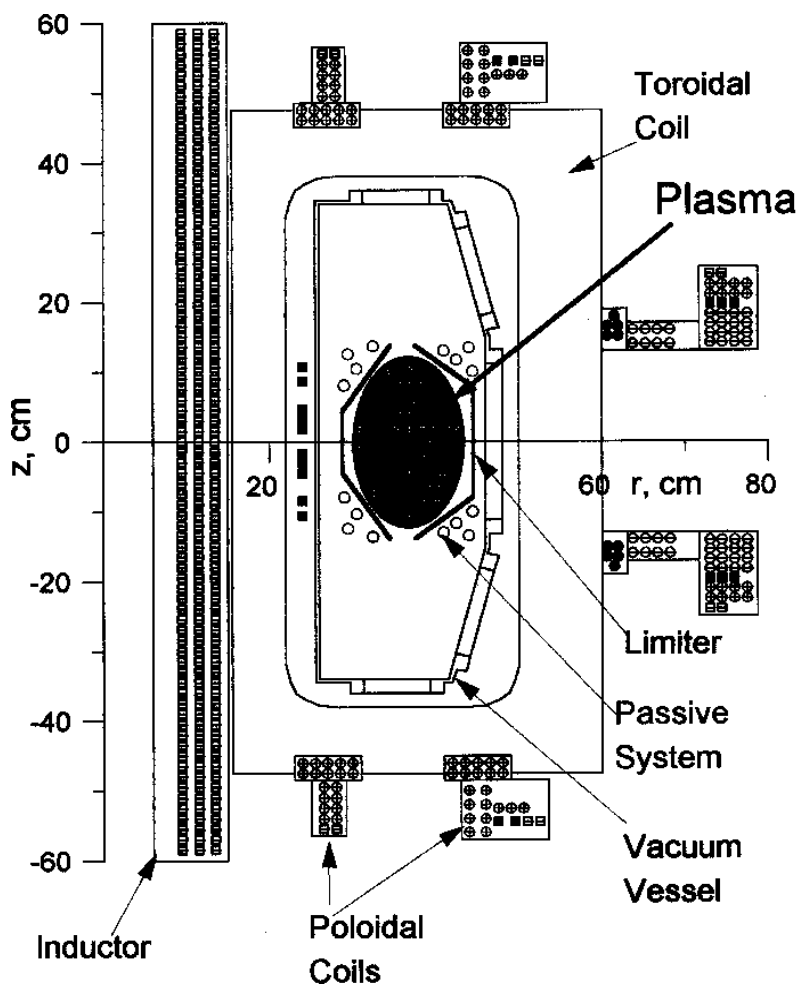

Figure 1. Damavand Tokamak cross-section. 


\begin{tabular}{|l|l|l|}
\hline Parameters & Max. Values & Typical Values \\
\hline Major radius of the torus & $R_{0}=36 \mathrm{~cm}$ & \\
Transversal cross-section & $2 \mathrm{a} / 2 \mathrm{~b}=20 / 56 \mathrm{~cm}$ & \\
Toroidal field & $B_{t}=1.2 \mathrm{~T}$ & $1.0 \mathrm{~T}$ \\
Discharge duration & $t>15 \mathrm{~ms}$ & $50 \mathrm{~ms}$ \\
Plasma density & $n(0)=3 \times 10^{19} \mathrm{~m}^{-3}$ & $2 \times 10^{19} \mathrm{~cm}^{-3}$ \\
Electron temperature & $\mathrm{Te}(0)=300 \mathrm{eV}$ & $280 \mathrm{eV}$ \\
Ion temperature & $\mathrm{Ti}(0)=150 \mathrm{eV}$ & $110 \mathrm{eV}$ \\
Plasma current & $I_{p} \sim 35-40 \mathrm{KA}$ & $35 \mathrm{KA}$ \\
\hline
\end{tabular}

Table 1. DAMAVAND Parameters.

This system can be represented by a simple set of first-order differential equations for the currents in the circuit elements, augmented by the equation of motion for the plasma column [8],

$$
\begin{gathered}
\frac{d}{d t}(\mathbf{M} \cdot \mathbf{I})+\mathbf{R} \cdot \mathbf{I}=\mathbf{V} \\
\frac{m d v}{d t}=\sum(\text { radial forces })
\end{gathered}
$$

Here $\mathbf{V}$ is the vector of applied voltages, $\mathbf{M}$ is the mutual inductance matrix, $\mathbf{R}$ is the (diagonal) matrix of resistance for the circuit elements, and $\mathbf{I}$ is the vector of currents in the circuit elements. In Eq. (2), $v$ is the plasma column radial velocity and $m$ is the mass of the plasma column. Solving the above equations has derived a simple linearized plasma position model applicable near equilibrium and its Laplace transform block diagram is shown in Fig.2, where

$$
\Delta_{H}(s)=G_{p}(s) \cdot I_{c}(s)+G_{1}(s) \cdot\left(\beta_{p}+\frac{l_{i}}{2}\right)(s)
$$

In this equation $s$, is the Laplace transform operator, $I_{c}(s)$ is the control coil current Laplace transform, $\beta_{p}$ is the poloidal beta, $l_{i}$ is the plasma internal inductance, and $G_{p}(s)$ and $G_{1}(s)$ are the plasma position and disturbance Laplace transfer functions which have been described in detail in ref. [4]. The parameters of these transfer functions are the complex functions of: 1) the electrical characteristics and geometry of the windings, vacuum vessel, and interacting electric circuits, and 2) plasma parameters such as current, major radius, and minor radius. The two most important parameters disturbing the plasma position in Damavand tokamak are the plasma temperature $(\mathrm{T})$ and density (n) fluctuations, which is included in the $\left(\beta_{p}+l_{i} / 2\right)$ parameter. In this model control coil current $I_{c}(t)$ has been chosen as control input because by varying this current the magnetic field in the plasma area can be easily modified and the plasma is kept in the desired location.

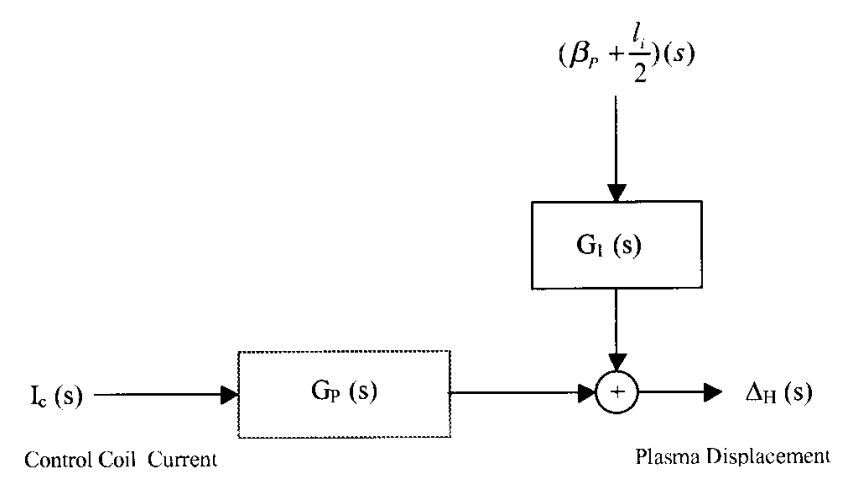

Figure 2. Plasma position Laplace transform block diagram.

\section{Digital Control System}

\section{III.1 Introduction}

The Damavand digital control system block diagram is shown in Fig. 3. The heart of this system is a DSP board, based on a high speed (12.5 MIPS) TMS320C25 chip [9]. It is used for the implementation of a digital control algorithm; it receives error signal e $(\mathrm{k})$ and generates the control output $u(k)$, which activates the control coil current. The TMS 320 family of processors is suitable for implementation of digital filter [9], [10]. They have the power for implementing a pole and zero of a digital filter with only two instructions. Due to fixed-point representation of numbers by the DSP, overflow may occur. Appropriate scaling becomes important to avoid this problem. The control coil current $I_{c}(t)$, is generated by a thyristor control circuit with the maximum time constant of $200 \mu \mathrm{s}$. Finally, the plasma position will be monitored and processed using a plasma position-processing unit. The other tokamak signals, which are required for plasma modeling and control, are acquired using a CAMAC based interface (IEEE-583) data acquisition system (Fig. 3). 


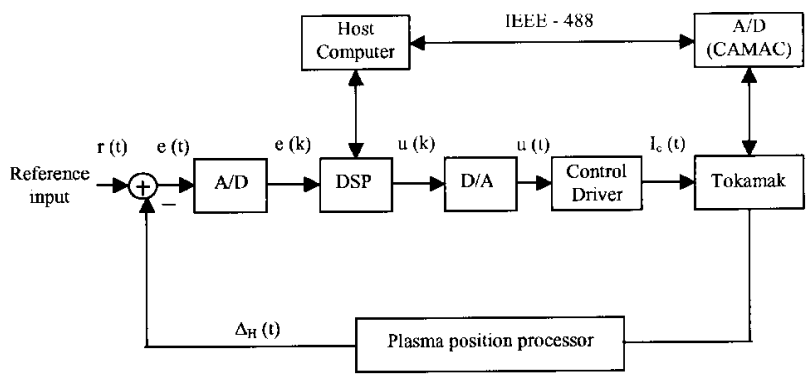

Figure 3. Plasma position digital control system.

\section{III.2 Digital Control System Analysis}

One of the most widely used control algorithms is the proportional-integral-derivative (PID). The digital PID control algorithm implemented for this work is used in the rectangular approximation [11], $\frac{T}{(z-1)}$ for the integral operation $(1 / s)$, and the backward difference approximation $\left(\frac{z-1}{T \cdot z}\right)$ for the derivative operation $(s)$, where $z=e^{s T}$ and $T$ is the sampling period. It should be realized that as long the sampling frequency is much bigger than the system bandwidth the above approximation holds. A digital PID controller algorithm using the above approximation can be written in the form of a recursive difference equation [9] as

$$
u(k)=u(k-1)+k_{0} e(k)+k_{1} e(k-1)+k_{2} e(k-2) .
$$

Where $e(k)$ and $u(k)$ are the controller input and output and,

$$
\begin{gathered}
k_{0}=k_{p}\left(1+\frac{T_{d}}{T}\right), \\
k_{1}=k_{p}\left(-1-2 \frac{T_{d}}{T}+\frac{T}{T_{i}}\right), \\
k_{2}=k_{p}\left(\frac{T_{d}}{T}\right) .
\end{gathered}
$$

Where $K_{p}, T_{i}$ and $T_{d}$ are the proportional gain, integral and derivative time constant of the equivalent analog PID controller. The DSP can execute the Equation (4) within $1.3 \mu \mathrm{s}$ and the time required for other tasks such as executing the timing routine, interrupt request handling and scaling numbers is $13 \mu \mathrm{s}$. The output from this calculation $u(k)$ can be fairly noisy, but the control coil current driver circuit with the 200 $\mu$ s time constant acts as a good low-pass filter.

The $A / D$ converter is modeled as an ideal impulse sampler combined with a finite time delay $t_{A / D}$ to account for the conversion time and is described by

$$
G_{A / D}(s)=K_{A / D} e^{-t_{A / D} s}
$$

Where $K_{A / D}$ is the $A / D$ gain. Finally, for simplicity $D / A$ converter is modeled as a zero- order hold device [11] and its Laplace transfer function is described as

$$
G_{D / A}(s)=K_{D / A} \frac{1-e^{-T s}}{s}
$$

Where $T$ and $K_{D / A}$ are the sampling period and converter gain, respectively.

\section{III.3 Digital Controller Performance}

The plasma position control system of Damavand tokamak based on a digital PID algorithm has been simulated by computer and is compared with the DSP. This simulation shows that the performance of the DSP, operating on 16 Bit fixed- point arithmetic is in close agreement with the computer, which uses double precision floating- point data. This is obviously due to the fact that the sampling rate is much higher than the system bandwidth.

The primary measure of the performance of the controller is its ability to control the plasma position when it is subject to disturbances. Fig. 4 shows the plasma position evolution with and without controller in a 10 $\mathrm{mm}$ step-wise disturbance at the instant of time $\mathrm{t}=8$ ms.

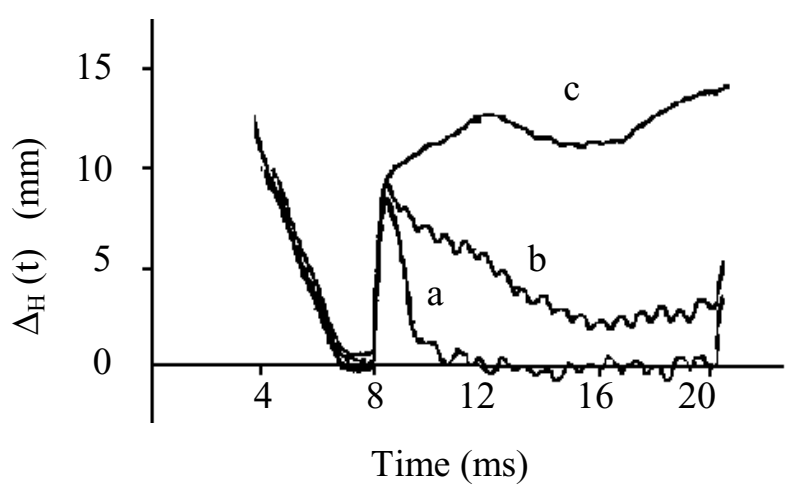

Figure 4. Plasma position control system step response: a) Digital controller, b) Analog controller and c) Without controller.

Because of the small current in build-up phase $(t<$ $8 \mathrm{~ms}$ ), the plasma position is very sensitive to the stray magnetic field. Also, due to the circuit limitation at low plasma current the plasma position measurement is not very accurate and the position is controlled by preprogrammed magnetic field. As it can be seen from Fig. 4b using the analog controller, the plasma has not returned to the center of the chamber $\left(\Delta_{H}=0\right)$ later in the discharge. The reason is that the analog controller gains were tuned for the steady state region of the plasma while for the last stage in the discharge, because of the changes in the plasma current, density and temperature, the parameters of the plasma model deviated from their steady-state value and the controller 
gains should have been changed accordingly during discharge. This was not convenient with the analog controller and has been replaced by digital controller.

\section{Conclusion}

When model parameters are changing due to the plasma parameter variations, the controller gains should be changed during the discharge. Since analog controller was not flexible it was replaced with a digital controller. A digital control system based on TMS320C25 microprocessor has been designed and built. The results show the digital signal processor performance is satisfactory as long as the sampling frequency is much larger than the bandwidth of the system which includes DSP and its associated circuitry delay time, thyristor current control circuit, control coil, vacuum vessel, and plasma position measurement unit time constants.

\section{References}

[1] J. Wesson, Tokamak, Oxford Science Publication, (1987).

[2] J. R. Ulgum "Digital Feedback control for TEXT Upgrade" Fusion Research Center, Austin, Texas FRCR\#412 (1992).

[3] J.B. Lister, F. Hofmann, J.M. Moret, M.J. Dutch, D. Fasel, A. Favre, P.F. Isoz, B. Marietz, P. Marmillod,
Y. Martin, A. Perez, and D.J. Ward, "The Control of TCV Plasmas" LRP518/95, July 1996.

[4] M. Emami, H. C. Wood, A. Hirose, "A controller for plasma motion in a tokamak based on model estimation", IEEE Transactions on Industrial electronics, Vol.37, 317 (1990).

[5] Y. Mizuno, H. Muramatsu, T. Aoki and T. Sometani, "Feedback position control apparatus of tokamak plasma horizontal position with a digital drive", Electrical Engineering in Japan Vol. 130, 26 (2000).

[6] A. Coutlis, J.N. Limebeer, J.P. Wainwright, J.B. Lister, and P. Vyas, "Frequency response identification of dynamics of a tokamak plasma" IEEE Transactions on Control System Technology, Vol.8, 646 (2000).

[7] R. Amrollahi, A.V. Bortnikov, N.N. Brevnov, Yu.V. Gott, and V.A. Shurygin, "Experimental Study of the Pre-Disruption in the Damavand Tokamak", Plasma Physics Reports, Vol. 23, 561 (1997).

[8] E.A. Lazarus, G.H. Neilson, "Solutions to the tokamak circuit equations with force balance for a massless plasma", Nuclear Fusion, Vol. 27, 383 (1987).

[9] TMS320C2X Fixed-Point User's Guide (Texas Instrument SPRU014C).

[10] Hardware Interfacing to the TMS320C2X (Texas Instrument SPRA014B).

[11] Digital Control Systems, C. Kuo, HRW series in Electrical and Computer Engineering, ISBN 0-03-057568-0. 